\title{
Effect of phase-transformation on mechanical behavior of dual-phase steel plate
}

\author{
H.Q. Liu, C. Zhu and G.C. Li \\ Institute of Mechanics, Academia Sinica, Beijing, 100080, People's Republic of China
}

\begin{abstract}
The mechanical behavior of dual phase steel plates is affected by internal stresses created during martensite transformation. Analytical modelling of this effect is made by considering a unit cell made of martensite inclusion in a ferrite matrix. A large strain finite element analysis is then performed to obtain the plane stress deformation state. Displayed numerically are the development of the plastic zone and distribution of local state of stress and strain. Studied also are the shape configuration of the martensite (hard-phase) that influences the interfacial condition as related to stress transmission and damage. Internal stresses are found to enhance the global flow stress after yield initiation in the ferrite matrix. Good agreement is obtained between the analytical results and experimental observations.
\end{abstract}

\section{Introduction}

Two-phase materials with a soft and hard constituent are desirable as their combination could enhance the strength and ductility. The attainment of optimum performance for a given application entails many considerations. Among them are the size and distribution of the structural constituents that depend on the heat treatment and manufacturing process to arrive at the desired geometric configuration. Dual-phase steel plates are widely used because of their versatility in engineering application. One of the important factors that govern their mechanical behavior is the internal stresses as induced by martensite transformation. An understanding of this influence would no doubt contribute to better optimization of the controlling processing variables.

Past attempt [1] has been made to analyze the mechanical behavior of martensite/ferrite steel plates where martensite islands are approximated as a single equivalent sphere embedded in an infinite ferrite matrix. Interaction between the hard particles is thus neglected in addition to assuming small strains. Since the dual-phase steel plate possesses good ductility and could sustain large deformation, it would be prudent to include such an effect in the analysis. This has been done in [2] where both phases of the material could undergo plastic deformation. Considered in [3] is also the average internal stresses in each phase of the plastically deformed constituents.

A more detailed analysis is made on a twophase steel plate in this work whereby large strain is invoked. The martensite islands are assumed to be periodically distributed such that it suffices to consider a unit cell consisting of a circular (or elliptic) inclusion in a ferrite matrix under plane stress. Uniform displacement boundary conditions are applied to the cell wall. The proposed model is adequate for investigating the effect of internal stresses arising from phase transformation and of martensite inclusion shape on the mechanical behavior of dual-phase steel plates. Abrupt geometric variations along the interfaces of the martensites are not considered.

\section{Analytical modelling}

Consider a two-phase material that contains periodic arrays of circular inclusions in both the $x_{1}$ and $x_{2}$ direction. Because of symmetry, it suffices to consider a cell made of martensite inclusion with radius $r_{0}$ in a ferrite matrix. Onequarter of the cell configuration is shown in Fig. 1 where unity is taken for its width and height. More specifically, the martensite volume fraction is taken as $18 \%$ and $r_{0}$ is normalized to be $48 \%$ of the half-width of the cell. 


\subsection{Proportional straining}

Let $u$ and $v$ be the $x_{1}$ - and $x_{2}$-component of the displacement, respectively. They are normalized with reference to half-width of the cell. Proportional straining is assumed such that displacement condition on the cell boundary satisfies the relation:

$$
\frac{\Delta u}{1+u}=-\lambda \frac{\Delta v}{1+v}, \quad\left(\text { or } \dot{\bar{\epsilon}}_{1}=-\lambda \dot{\bar{\epsilon}}_{2}\right)
$$

The quantity $\lambda$ plays the role of a proportionality factor. Assumed throughout this analysis is a state of plane stress. Here, dot represents time differentiation. Alternatively, the strain increments may be written as

$\mathrm{d} \vec{\epsilon}_{1}=\frac{\Delta u}{1+u}, \quad \mathrm{~d} \bar{\epsilon}_{2}=\frac{\Delta v}{1+v}$

such that

$\bar{\epsilon}_{1}=\ln (1+u), \quad \bar{\epsilon}_{2}=\ln (1+v)$

The bar on the strain components denotes they refer to the global continuum system while local strains are referred to simply as $\epsilon_{j}$ without the bar.

\subsection{Principal stresses}

The principal stresses $\sigma_{1}$ and $\sigma_{2}$ normalized with the Young's modulus are denoted as $\bar{\sigma}_{1}$ and

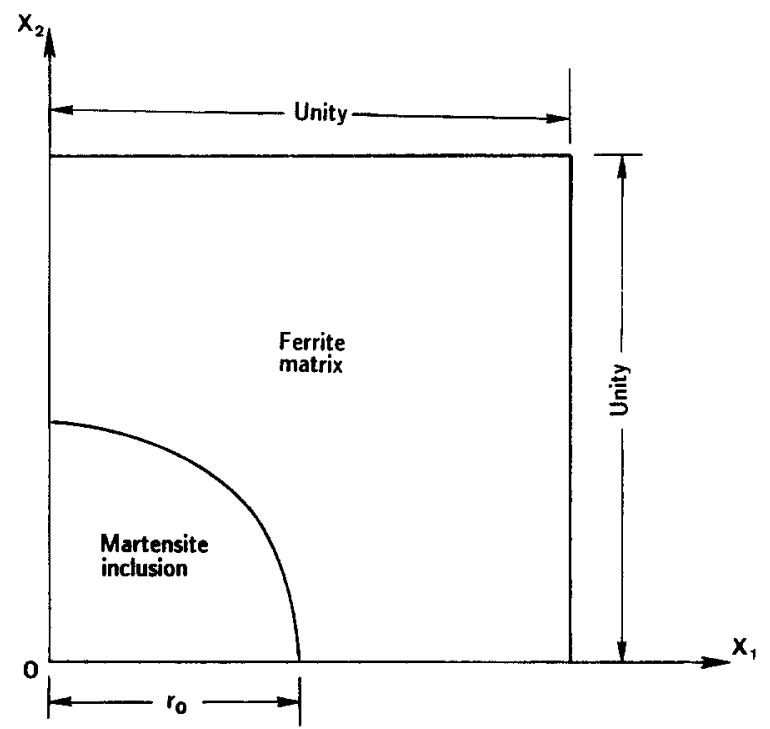

Fig. 1. A quadrant of cell model. $\bar{\sigma}_{2}$, respectively. Again, the bar refers to global quantities. They reflect the response of all the nodal forces $F_{1}^{i}$ and $F_{2}^{i}$ along the cell boundaries and can be expressed as

$\bar{\sigma}_{1}=\sum_{i} \frac{F_{1}^{i}}{(1+v)(1+w)}$, for $x_{1}=1+u$

and

$\bar{\sigma}_{2}=\sum_{i} \frac{F_{2}^{i}}{(1+u)(1+w)}$, for $x_{2}=1+v$

Here, $i$ denotes location of a particular nodal point on the cell boundary. In eqs. (4) and (5), w is the change in plate thickness averaged over the area of the cell; it is also normalized with reference to the undeformed plate thickness. Stress symmetry with respect to the axes o $x_{1}$ and $o x_{2}$ in Fig. 1 requires that nodal force rate to vanish on $x_{1}=1+u$ and $x_{2}=1+v$ as follows:

$\dot{F}_{2}^{i}=0 \quad$ at $x_{1}=1+u$;

$\dot{F}_{1}^{i}=0$ at $x_{2}=1+v$

\subsection{Finite element procedure}

An updated Lagrangian finite element formulation [6,7] is employed that applies the isotropic Prandtl-Reuss constitutive relation. An incremental solution involving $10^{3}$ steps is obtained by taking the elongation $v$ as the generalized time variable $t$. The relation between the nodal force $\{\boldsymbol{F}\}^{\mathrm{e}}$ caused by the expansion expressed by the average $\epsilon_{\mathrm{m}}^{*}$ a result of martensite transformation is given by

$$
\{\boldsymbol{F}\}^{\mathrm{e}}=[B]^{\mathrm{T}}[D]\left\{\boldsymbol{\epsilon}_{\mathrm{m}}^{*}\right\} h A
$$

where $h$ is the plate thickness and $A$ the element area. The geometric matrix $[B]$ is related to the derivatives of the shape functions and $[D]$ is the elastic stiffness matrix of the material. The superscript $T$ stands for transpose of the matrix and $e$ stands for an element that is triangle in shape with constant strain. The quadrant in Fig. 1 is subdivided into 1066 constant-strain triangular elements with 755 nodes. Equation (7) is enforced on the martensite portion in the cell in an incremental manner while the boundary lines are kept straight and fixed. The average expansion strain $\epsilon_{\mathrm{m}}^{*}$ will be determined experimentally. 


\section{Experimental consideration}

The material used in this study is a vacuum smelted low carbon manganese steel, the chemical composition of which is given by weight as outlined in Table 1. The specimens are annealed at $1280^{\circ} \mathrm{C}$ for 5 hours and then heated to the $\alpha$ and $\gamma$ phase region at $760^{\circ} \mathrm{C}$ for 20 minutes followed by quenching in water. The resulting microstructure contains martensite islands dispersed over ferrite grains. Measured by a Cambridge Q-520 image analyzer, the average diameter of the ferrite and martensite is, respectively, $48 \mu \mathrm{m}$ and $46 \mu \mathrm{m}$ with an $18 \%$ volume fraction of martensite.

\subsection{Mechanical properties}

A schematic of the tensile specimen is given in Fig. 2 with a gauge length of $6.5 \mathrm{~mm}$ along which the elongation is measured for each increment of loading. The true axial strain $\bar{\epsilon}_{2}$ (or $\bar{\epsilon}$ ) and true axial stress $\bar{\sigma}_{2}$ (or $\bar{\sigma}$ ) are obtained as displayed in Fig. 3 for the dual-phase steel. The yield strength $\sigma_{\mathrm{y}}$ and tensile strength $\sigma_{\mathrm{t}}$ are, respectively, 370 $\mathrm{MPa}$ and $650 \mathrm{MPa}$. Figure 4 gives the stress and strain behavior of the ferrite. Using a superscript $o$ to denote room temperature, the yield strength of ferrite is equal to that of the dual-phase steel, i.e., $\sigma_{\mathrm{yf}}^{0}=\sigma_{\mathrm{y}}=370 \mathrm{MPa}$. At the phase-transition temperature $350^{\circ} \mathrm{C}$, the yield strength $\sigma_{\mathrm{yf}}^{\mathrm{p}}$ is 185 $\mathrm{MPa}$. The corresponding yield stresses for martensite are $\sigma_{\mathrm{ym}}^{0}=1400 \mathrm{MPa}$ at room temperature and $\sigma_{\mathrm{ym}}^{\mathrm{p}}=930 \mathrm{MPa}$ at $350^{\circ} \mathrm{C}$.

The dual-phase steel exhibit anisotropy [5] in deformation when the nominal axial strain exceeds 0.1 . Shear-band localization would then occur. Prior to this stage, the strains along the specimen width and thickness are almost the same as axial strains proceeds. Triaxiality tends to increase as the thickness reduction becomes more appreciable in comparison with the change along the specimen width. Isotropic modelling of the constitutive relation used in the calculation holds

Table 1

Chemical composition (wt\%) of low carbon manganese steel

\begin{tabular}{llllll}
\hline $\mathrm{C}$ & $\mathrm{Mn}$ & $\mathrm{Si}$ & $\mathrm{P}$ & $\mathrm{S}$ & $\mathrm{Fe}$ \\
\hline 0.08 & 1.50 & 0.30 & 0.08 & 0.010 & balance \\
\hline
\end{tabular}

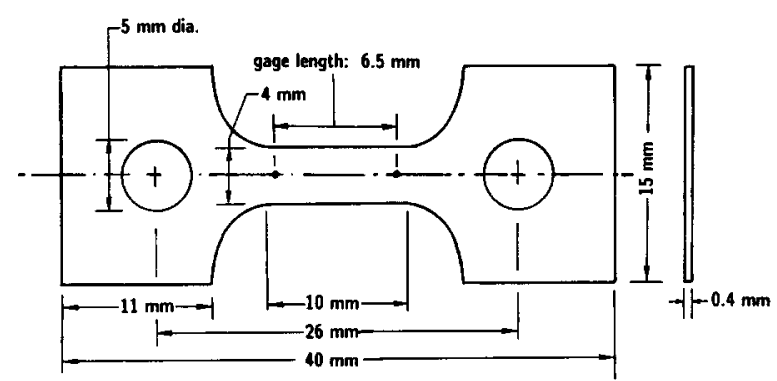

Fig. 2. Uniaxial tensile specimen.

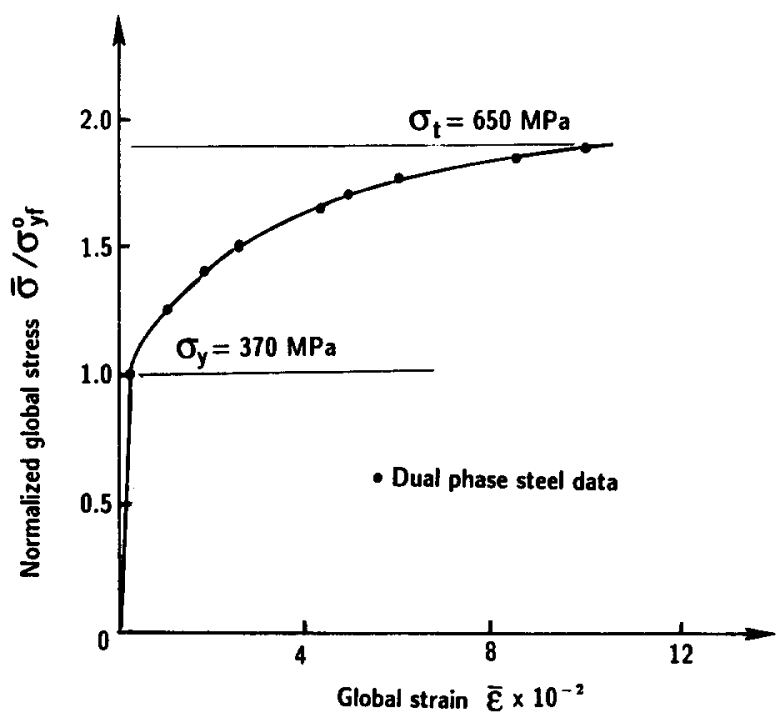

Fig. 3. Uniaxial tensile data for dual-phase steel.

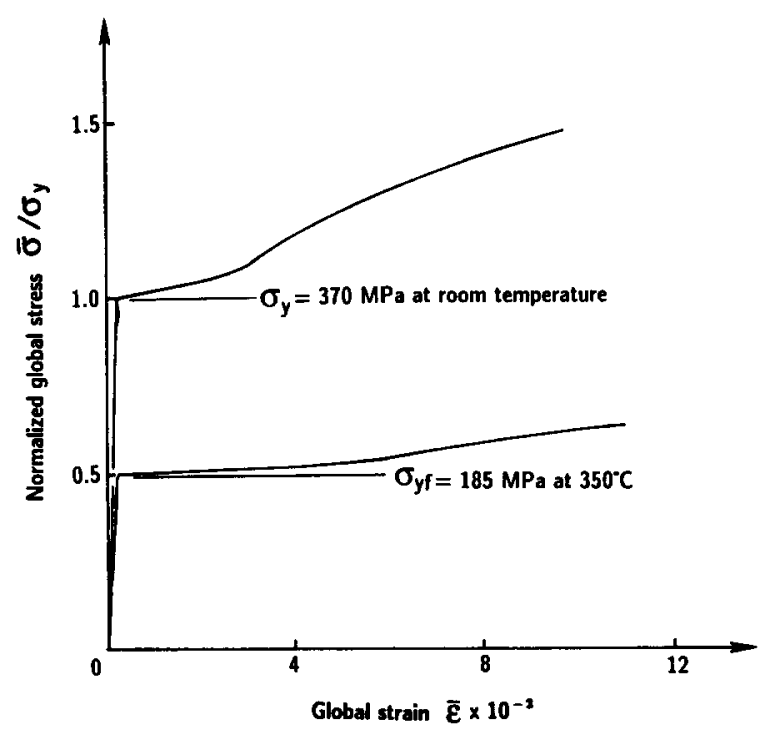

Fig. 4. Uniaxial tensile data of ferrite. 


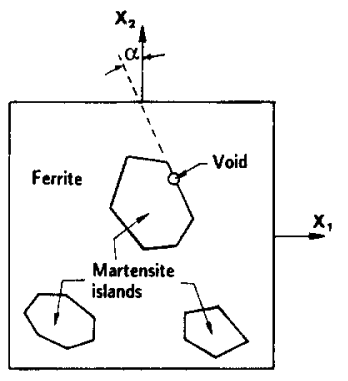

(a) Interface inclination

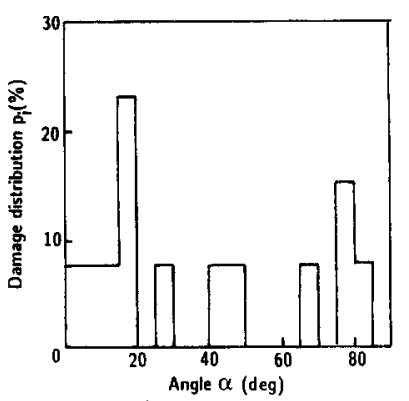

(b) Nominal strain 0.102
Fig. 5. Schematic of martensite islands in ferrite and damage distribution with reference to orientation of interfacial plane.

before the material local damage sets in the necked region.

The phase transformation of martensite occurs at the temperature of $350^{\circ}$ or a little higher. The average expansion strain $\epsilon_{\mathrm{m}}^{*}$ is estimated from a metallurgic formula [1]:

$\epsilon_{\mathrm{m}}^{*}=0.016-0.0017 C$

where $\mathrm{C}$ denotes the wt $(\%)$ of carbon component in the martensite. For the present problem, eq. (8) gives $\epsilon_{\mathrm{m}}^{*}=0.0155$. This value will be used in eq. (7) for the finite element calculation.

\subsection{Damage observation}

A Hitachi S-570 Scanning electron microscope (SEM) is used to examine the in-situ damage of the uniaxial tensile specimen in Fig. 2. SEM photographs are taken at several time intervals for recording the different stages of damage. Development of voids can be observed along the martensite / ferrite interface. Corner points of the martensite inclusion tend to enhance void nucleation. Figure 5(a) illustrate the polygon-like martensite islands with interfacial lines, the inclination of which, say $\alpha$, would vary from $0^{\circ}$ to $90^{\circ}$. Eighteen equal angular sections each with a subtended angle of $5^{\circ}$ are taken as reference for the measurements. The proportion of interfacial damage within each angular section is accounted for by the distribution

$p_{i}=\frac{n_{i}}{N}$

The total number of interfacial lines with voids is $N$ and $n_{i}$ is the number of lines within the $i$ th angular section. Hence, $p_{i}$ would provide a measure of void damage along the interfacial lines. A plot of $p_{i}$ against $\alpha$ can be found in Fig. 5(b) for a nominal axial strain of 0.102 . With $x_{2}$ and $x_{1}$ along the axial and width direction of the specimen in Fig. 2, small $\alpha$, say $\alpha<20^{\circ}$, would correspond to interfacial lines close to the $x_{2}$-axis and large $\alpha$, say $\alpha>75^{\circ}$ would correspond to planes close to the $x_{1}$-axis. The two peaks in Fig. 5(b) refer to interfacial damage due to the action of tangential shear $\left(\alpha<20^{\circ}\right)$ and normal stress $(\alpha>$ $\left.75^{\circ}\right)$.

\section{Discussion of results}

Analytical results are obtained for proportional straining such that eq. (1) is satisfied at all time as extension in the axial or $x_{2}$-direction is increased. This is accomplished by varying the choice of the parameter $\lambda$ such that a uniaxial state of stress prevails with vanishing $\bar{\sigma}_{1}$ as given by eq. (4). Under plane stress, $\bar{\sigma}_{3}$ is set to zero at the start. Only the global axial stress $\bar{\sigma}_{2}$ would prevail. The Young's modulus and Poisson's ratio for both the martensite and ferrite are taken as $E=207 \mathrm{GPa}$ and $\nu=0.3$, respectively. Four hundred incremental steps of computation were required to reach the martensite expansion level of $\epsilon_{\mathrm{m}}^{*}=0.0155$. During the stage of phase transformation, the martensites remained elastic in compression while plastic zones developed in the ferrite around the martensites. Void damage along the interface starts as $\mathrm{v}$ approaches 0.102 . The local stress and strain distribution provides insight into the mechanical behavior of the dualphase steel material.

\subsection{Internal stress from phase transformation}

Phase transformation induces internal stresses in the two-phase material from which the effective stress $\sigma_{\mathrm{e}}$ and mean stress $\sigma_{\mathrm{m}}$ can be computed to reflect the extent of distortion and dilatation. Figure 6(a) gives the numerical values of

$\sigma_{\mathrm{e}}=\left(\sigma_{11}^{2}-\sigma_{11} \sigma_{22}+\sigma_{22}^{2}+3 \sigma_{12}^{2}\right)^{1 / 2}$

as a function of the distance $r$ normalized against the one-half cell width in Fig. 1. Value of $\sigma_{\mathrm{e}}$ at $\sigma_{\mathrm{e}} / \sigma_{\mathrm{yf}}=1$ is $185 \mathrm{MPa}$ for $r$ from 0.6 to 0.8 as all 

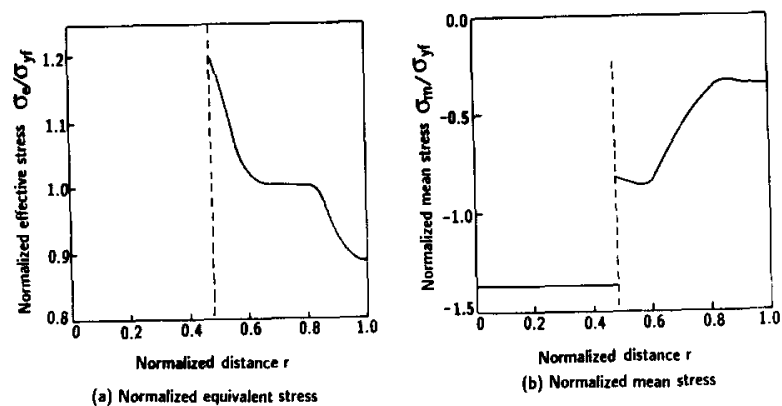

Fig. 6. Variations of local effective and mean stress in cell.

data are referred to $\sigma_{\mathrm{yf}}=185 \mathrm{MPa}$. The effective stress in the ferrite is largest at $r=0.48$ and decreases rapidly with increasing distance away from the interface. The variations of the mean stress

$\sigma_{\mathrm{m}}=\frac{1}{3}\left(\sigma_{11}+\sigma_{22}\right), \quad \sigma_{33}=0$

with $r$ are exhibited in Fig. 6(b). Note that compressive biaxial stress state has a constant intensity in the martensite and decreases in magnitude in the ferrite with a discontinuity at the interface.

\subsection{Post yielding}

Transformation induced yielding occurs in a layer around the martensite while the intensity of yielding in the ferrite increases as the interface is approached. This is illustrated in Fig. 7 where $\sigma_{\mathrm{e}} / \sigma_{\mathrm{yf}}^{0}$ (with $\sigma_{\mathrm{yf}}^{0}=370 \mathrm{MPa}$ ) is plotted against $r$.

Further increase in uniaxial tensile load tends to intensify yielding and spread the post-yielding zone over a larger portion of the ferrite. Increase in the size of this zone is shown in Fig. 8(a) for $v=2 \times 10^{-3}$, Fig. $8(\mathrm{~b})$ for $v=2.9 \times 10^{-3}$ and Fig. $8(\mathrm{c})$ for $v=3.6 \times 10^{-3}$.

\subsection{Large deformation}

Illustrated in Fig. 9 are the influence of large deformation and internal stress on the mechanical behavior of the two-phase steel. As anticipated, the difference between large and small deformation solution becomes significant as the strain is increased. Exclusion of internal stress tends to underestimate the stress level at a given strain beyond yield. Comparing with the uniaxial experimental data in Fig. 3, all three curves in Fig. 9 give lower predictions.

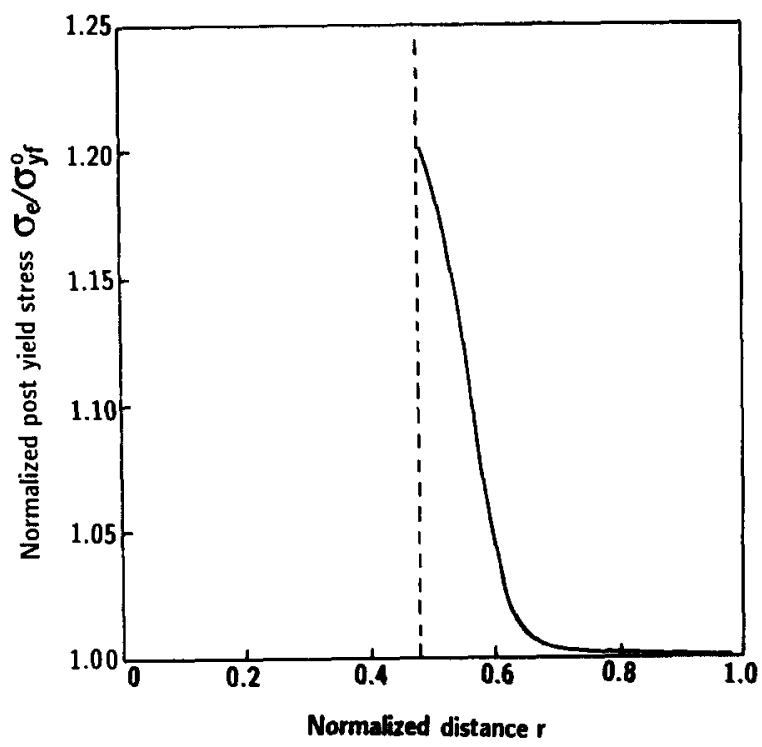

Fig. 7. Distribution of post yield stress in ferrite.
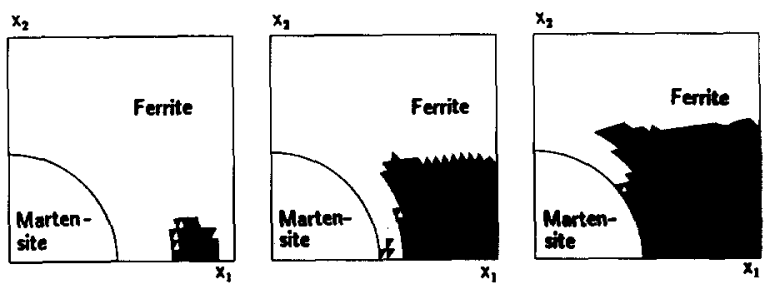

(a) $v=2 \times 10^{-3}$

(b) $v=2.9 \times 10^{-3}$

(c) $v=3.6 \times 10^{-3}$

Fig. 8. Post yield zones for increasing strain.

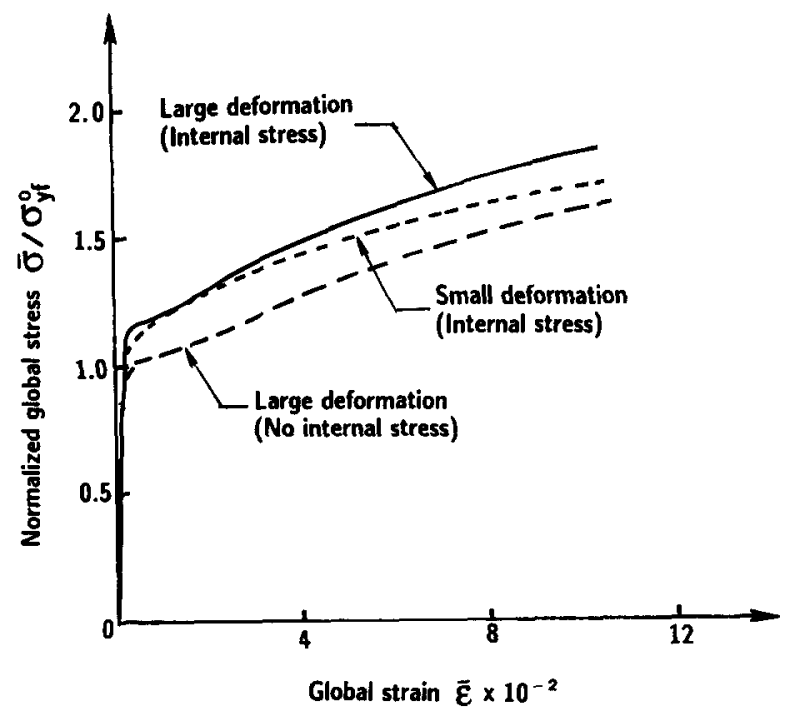

Fig. 9. Calculated stress and strain behavior of dual-phase steel. 


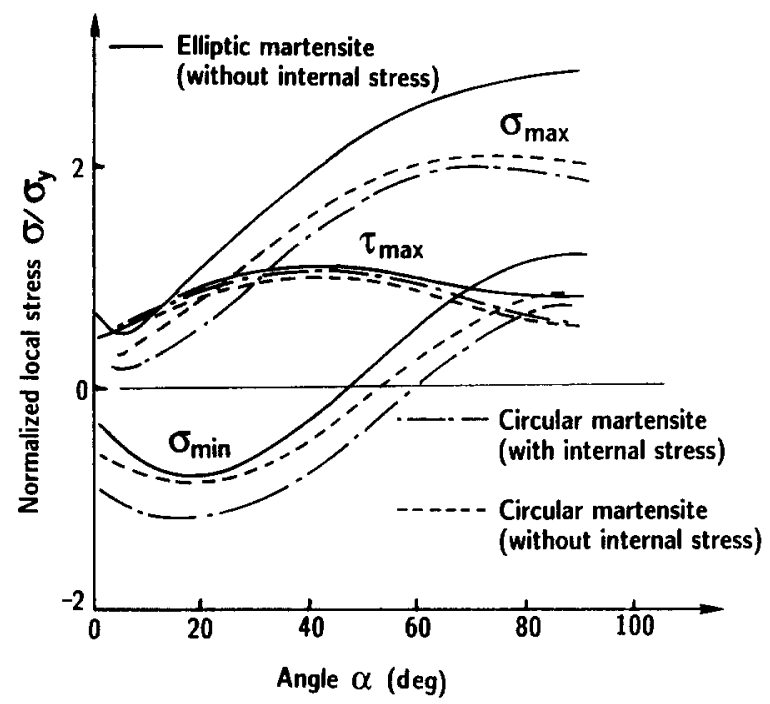

Fig. 10. Principal normal and maximum shear stresses along interface at $v=0.102$.

\subsection{Interfacial stresses}

The interfacial principal stresses $\sigma_{\max }$ and $\sigma_{\min }$ and shear stress $\tau_{\max }$ would vary as the angle $\alpha$ between the load and interfacial plane is changed. Their variations are shown in Fig. 10 for $v=$ 0.102. For $\alpha$ less than $45^{\circ}, \sigma_{\max }$ is tensile and $\sigma_{\min }$ is compressive. This would enhance shear action. When both $\sigma_{\max }$ and $\sigma_{\min }$ are positive, shear tends to decrease.

\subsubsection{Internal stress}

Referring to the two sets of data for the circular martensite inclusion with and without internal stress, both $\sigma_{\max }$ and $\tau_{\max }$ are not affected. Significant difference occurs in $\sigma_{\min }$ and should be accounted for when evaluating interfacial damage.

\subsubsection{Inclusion shape}

The solid curves in Fig. 10 refer to ellipticallyshape martensite inclusion with an aspect ratio of 2. Affected the most is $\sigma_{\max }$ as the angle $\alpha$ is increased. The general trend is that shear is enhanced for small $\alpha$ and extension for large $\alpha$. These features were reflected in Fig. 5(b) in relation to data on interfacial damage.

\subsection{Remarks}

Internal stress induced by martensite transformation in dual-phase steels can enhance the strength and ductility. Understanding gained from this analysis on interfacial damage provides insight into the failure of dual-phase materials.

\section{Acknowledgements}

This study was jointly supported by the National Natural Science Foundation of China (No. 19272066) and Chinese Academy of Sciences (KM85-33).

\section{References}

[1] T. Sakaki, K. Sugimoto and T. Fukuzato, Role of internal stress for continuous yielding of dual-phase steels, Acta Metall. 31 (10) 1737-1746 (1983).

[2] Y. Tomota and K. Kuroki, Tensile deformation of twoductile-phase alloy: Flow curves of $\alpha-\gamma \mathrm{Fe}-\mathrm{Cr}-\mathrm{Ni}$ alloys, Materials Science and Eng. 24, pp. 85-94 (1976).

[3] Y. Tomota, S. Nakamura and K. Kuroki, On the average internal stresses in each constituent phase in plastically deformed two-ductile-phase alloy, Materials Science and Eng. 46, 69-74 (1980).

[4] X.T. Sun, The Mechanical Properties of Metals, The Publication of Machinery Industry of China (1982) pp. 225-240 (in Chinese).

[5] T. Sakaki, K. Ohnuma, K. Sugimoto and Y. Ohtakara, Plastic anisotropy of dual-phase steels, Int. J. Plasticity 6, 591-613 (1990).

[6] R.M. McMeeking and J.R. Rice, Finite element formulations for problem of large elastic-plastic deformation, Int. J. Solids Struct. 11, 601-616 (1975).

[7] G.C. Li and I.C. Howard, The effect of strain softening in the matrix material during void growth, J. Mech. Phys. Solids 31, 85-102 (1983). 